\title{
Lesão por pressão: avaliação dos custos do tratamento em idosos atendidos em domicílio na saúde suplementar
}

\author{
Pressure injury: assessment of treatment costs in home-cared elderly in \\ supplemental health
}

\section{Daños por presión: evaluación de los gastos de asistencia domiciliar con ancianos en salud complementaria}

\author{
Vanessa Gomes Augusto ${ }^{1,2,3}$, Michele Pontes Moreira1,2,3, Solange Gurgel Alexandre ${ }^{4,5,6}$
}

\begin{abstract}
RESUMO
A lesão por pressão é um problema de saúde altamente complexo, multifatorial e de alto custo, sendo definida como qualquer lesão localizada na pele e/ou no tecido subjacente, normalmente sobre uma proeminência óssea. Objetivou-se avaliar a relação custo-benefício relacionada ao tratamento de lesões por pressão, com enfoque em idosos atendidos em domicílio na saúde suplementar. Estudo descritivo com abordagem quantitativa, envolvendo prontuários de idosos com diagnóstico de lesões por pressão, atendidos em uma instituição na modalidade autogestão, localizada em Fortaleza (CE). Os dados foram coletados mediante análise de relatórios contendo informações relacionadas ao tratamento, associando-os com os seus gastos financeiros. Os dados foram analisados estatisticamente e organizados em quatro planilhas que estão divididas por: período, procedimento, beneficiário e credenciado. Sabemos que a lesão por pressão interfere negativamente no bem-estar dos idosos, empobrecendo o seu viver. Dessa forma, é relevante o incentivo à prevenção e à construção de educação permanente aos cuidadores e familiares, por meio de estratégias de promoção de saúde realizadas pelos profissionais de Enfermagem.
\end{abstract}

DESCRITORES: Lesão por pressão. Análise de custos. Assistência domiciliar. Enfermagem. Estomaterapia.

\begin{abstract}
Pressure injury is a highly complex multifactorial and costly health problem, which is defined as any injury located on the skin and/or underlying tissue, usually over a bony prominence. The objective is to measure the cost and benefit relation regarding the treatment of pressure injuries, focused on the elderly who are treated at home in supplementary health. This is a descriptive study with a quantitative approach, involving medical records of elderly subjects diagnosed with pressure injury, cared for in an institution of self-management, located in Fortaleza (Ceará State, Brazil). Data were collected through the analysis of reports with information about the treatment by associating them with their financial costs. These data were statistically analyzed and organized in four worksheets that were divided into period, procedure, beneficiary, and certified subject. It is known that pressure injury negatively affects the welfare of the elderly, impoverishing their living. Thus, the support to prevention and development of the permanent education of caregivers and family is relevant, by means of health promotion strategies made by Nursing professionals.
\end{abstract}

DESCRIPTORS: Pressure injury. Cost analysis. Home nursing. Nursing. Stomatherapy.

${ }^{1}$ Caixa de Assistência dos Servidores Fazendários Estaduais (CAFAZ) - Fortaleza (CE), Brasil.

${ }^{2}$ Auditoria de Serviço Público e Privado do Centro de Qualificação e Ensino Profissional (CEQUALE) - Fortaleza (CE), Brasil.

3Pós-graduação em Estomaterapia na Universidade Estadual do Ceará (UECE) - Fortaleza (CE), Brasil.

Endereço para correspondência: Avenida Francisco Sá, 1.855, apto. 201 - Jacarecanga - CEP: 60010-450 - Fortaleza (CE), Brasil - E-mail: van_ga@hotmail.com ${ }^{4}$ Hospital Universitário Walter Cantídio - Fortaleza (CE), Brasil.

${ }^{5}$ Auditoria em Enfermagem do CEQUALE - Fortaleza (CE), Brasil.

¿UECE - Fortaleza (CE), Brasil.

Artigo recebido em: 04/04/2016 - Aceito para publicação em: 25/07/2016 


\section{RESUMEN}

Los daños por presión son un problema de salud muy complejo, multifactorial y con alto costo, y se definen como cualquier lesión localizada en la piel y/o en el tejido subyacente, generalmente sobre una prominencia ósea. Este estudio tuvo como objetivo evaluar el costo/beneficio relacionado con el tratamiento de los daños por presión, con un enfoque en ancianos tratados en sus hogares en la salud complementar. Estudio descriptivo con un enfoque cuantitativo, con la inclusión de los registros de pacientes ancianos con diagnóstico de los daños por presión, atendidos en una institución en la modalidad de autogestión, que se encuentra en Fortaleza (Ceará, Brasil). Los datos fueron recolectados a través del análisis de los informes que contienen datos relacionados con el tratamiento, asociándolos con sus gastos financieros. Los datos se analizaron y se organizaron en cuatro hojas que están divididas por: período, procedimiento, beneficiario y acreditado. Se sabe que los daños por presión afectan negativamente el bienestar de los ancianos, empobreciendo su vivir. Por lo tanto, es importante fomentar la prevención y la construcción de la educación permanente para cuidadores y familiares a través de estrategias de promoción de la salud llevadas a cabo por los profesionales de Enfermería.

DESCRIPTORES: Daños por presión. Análisis de costo. Atención domiciliaria de salud. Enfermería. Estomaterapia.

\section{INTRODUÇÃO}

O envelhecimento populacional é um fenômeno conhecido mundialmente. O Brasil destaca-se por viver esse processo de forma acelerada na última década. Entre 1980 e 2009, a expectativa de vida dos brasileiros aumentou mais de 10 anos, passando de 62,6 para 73,2 anos. Além disso, estima-se que, em 2050, mais de $15,0 \%$ da população brasileira terá 70 anos ou mais ${ }^{1}$.

A pele apresenta sinais de involução com o avançar da idade, que se tornam evidentes em torno dos 65 anos, quando os componentes das camadas sofrem alterações estruturais, numéricas e funcionais, sendo o envelhecimento um dos fatores de risco para a formação de lesão por pressão (LP) ${ }^{2}$.

A LP é um problema de saúde pública altamente complexo, multifatorial e de alto custo, sendo definida como lesões de pele e/ou tecidos subjacentes, localizadas normalmente sobre uma proeminência óssea, resultantes da pressão ou da combinação entre esta e forças de cisalhamento. Podem ser resultantes de eventos indesejados ocorridos durante a hospitalização, os quais refletem de forma indireta a qualidade do cuidado prestado, sendo um dos maiores desafios para os profissionais, principalmente de Enfermagem, pelos encargos econômicos que acarretam e pela diminuição na qualidade de vida dos clientes e seus familiares ${ }^{3,4}$.

São comumente encontradas em áreas suscetíveis à distribuição desigual de peso ou vulneráveis a pressões externas persistentes, como as regiões sacral, coccígea, isquiática, trocantérica e calcânea. São classificadas em quatro estágios, de acordo com a perda tecidual decorrente ${ }^{5}$.

A LP é um sério problema que afeta aproximadamente 9,0\% de todos os pacientes hospitalizados, sobretudo os idosos, e 23,0\% dos acamados com cuidados domiciliares. O seu desenvolvimento concorre para o aumento dos custos de saúde com o idoso, interferindo negativamente em seu bem-estar físico, mental e espiritual, restringindo-o ao leito e empobrecendo seu viver, independentemente do contexto em que esteja inserido ${ }^{6}$.

Além de ser um alvo de grande preocupação para os serviços de saúde, a ocorrência da LP também ocasiona grande impacto ao indivíduo, à família, à sociedade e aos profissionais de Enfermagem, acarretando graves repercussões sanitárias, econômicas, éticas e legais. É válido ressaltar que alguns autores consideram o aparecimento das LP como iatrogenias secundárias a falhas no processo de cuidar, em razão de existir, na atualidade, inúmeras medidas preventivas para o problema, bem como uma variedade de inovações tecnológicas para a sua terapêutica² ${ }^{2}$.

Apesar dos avanços no entendimento da etiopatogenia e da adoção predominante de medidas preventivas, estudos mostram que os índices de incidência e prevalência das LP permanecem elevados mundialmente.

É evidente a importância de reduzir a incidência de LP, considerado um importante indicador de qualidade assistencial, por intermédio da prevenção e identificação dos fatores de risco, o que pode ocorrer por meio da educação permanente da equipe multiprofissional, com uma prática baseada em evidências, permitindo analisar os casos quanto à sua distribuição, à vulnerabilidade dos pacientes e ao local em que as lesões são mais frequentes ${ }^{7}$.

Assim, é imprescindível que se investigue o nível de risco de pacientes em adquirir LP, bem como a prevalência destas e os custos atuais com curativos, de modo a melhorar os serviços de saúde e minimizar seus custos. Numa época marcada por 
profunda crise econômica, é essencial que os enfermeiros e demais profissionais de saúde compreendam a relevância dos custos econômicos associados a esta problemática ${ }^{8}$.

Apesar de resultados que apontam o montante gasto com o tratamento de LP, a compreensão acerca da natureza desses custos com aspectos subjetivos, como tempo de trabalho e número de horas destinadas aos cuidados rotineiros com LP, como a realização de curativos por profissionais de Enfermagem, não é de fácil entendimento9 .

Nesse contexto, é pertinente mencionar que a saúde suplementar, enquanto parte do mercado de assistência à saúde, está exposta a fortes influências dos aspectos envolvidos, principalmente no que concerne à sua dimensão econômica, que repercute em um interesse pela redução de custos e reorganização das modelagens de produção do cuidado, encontrando na lógica da assistência domiciliar (AD) uma possibilidade de racionalização de gastos e de encontrar alternativas viáveis para atender à demanda crescente ${ }^{10}$.

$\mathrm{O}$ crescimento universal da $\mathrm{AD}$ visa atender às necessidades da população, oriundas do envelhecimento populacional e consequente aumento da longevidade. Tendências epidemiológicas e demográficas têm sinalizado para um aumento das condições crônicas, o que vai gerar elevado custo em termos de sofrimento, incapacidades e perdas econômicas, tornando-se um dos maiores desafios do setor de saúde neste século ${ }^{11}$.

A AD tem como meta acompanhar e controlar em domicílio as condições de saúde de indivíduos com alguma alteração de saúde que os torne parcial ou totalmente dependentes. Tem como premissa a humanização da atenção à saúde, com o desenvolvimento de ações que proporcionem assistência integral à saúde de seus beneficiários assistidos, por meio de equipe multidisciplinar com habilidades específicas para essa prática.

Em geral, serviços de $\mathrm{AD}$ a idosos são considerados uma das estratégias mais importantes para o cuidado dessa população e uma das tendências do sistema de saúde ${ }^{12}$.

É importante refletir que a $\mathrm{AD}$ na saúde suplementar encontra-se em um campo de tensões: de um lado, a lógica de racionalização gerencial e financeira, representada pela redução de custos dos procedimentos; e do outro, a $\mathrm{AD}$, revelando-se como uma modalidade assistencial preferencial, seja por diminuir riscos, promover qualidade de vida ou aproximar o cuidado ao cotidiano das famílias ${ }^{10}$.

Enquanto parte dessa conjuntura, a auditoria é um sistema de caráter educativo e tem um papel fundamental de assessoramento na administração dos planos de saúde, com a finalidade de avaliar o padrão da assistência dos prestadores de serviço, apontando as distorções que precisam ser corrigidas e contribuindo com sugestões para melhorar a gestão e a qualidade do processo assistencial de acordo com os recursos financeiros disponíveis ${ }^{13}$.

Diante do exposto, é relevante equacionar a relação custo/benefício no processo de tratamento de idosos com LP, em domicílio, analisando e controlando os gastos financeiros - de forma especial aqueles relacionados à realização de curativos - e desenvolvendo estratégias de educação permanente, voltadas ao cuidador e à família. Essas estratégias devem ser eficazes para a prevenção desse agravo, objetivando estabelecer maior conforto ao associar condições favoráveis de bem-estar geral dos idosos à minoração dos custos ao convênio e baixos custos à excelência de qualidade no atendimento dos beneficiários.

Nesse contexto, objetivou-se avaliar a relação custo/ benefício relacionado ao tratamento da LP em idosos em atendimento domiciliar na saúde suplementar.

\section{MÉTODOS}

Trata-se de uma pesquisa de natureza descritiva, documental, com abordagem quantitativa, realizada em uma empresa de assistência médica, na modalidade autogestão, localizada na cidade de Fortaleza, no estado do Ceará. Fundada em 1991, a empresa presta serviços de saúde a seus beneficiários, funcionários de instituição pública da administração estadual e seus dependentes, sendo parte do setor suplementar de saúde e regulada pela Agência Nacional de Saúde Suplementar (ANS), tendo como missão garantir a excelência dos serviços em saúde, de forma humanizada.

A amostra do estudo foi composta por indivíduos com idade igual ou superior a 65 anos, de ambos os sexos, considerados idosos pelo Estatuto do Idoso - que preconiza e assegura direitos às pessoas com idade igual ou superior a 60 anos -, atendidos no Programa de Atendimento Domiciliar (PAD), com diagnóstico de LP, em qualquer categoria, no período compreendido entre 2011 e 2013, cujos dados relacionados constassem nos respectivos prontuários, arquivados na empresa.

Para iniciar a coleta de dados, obtivemos junto à empresa a assinatura do termo de fiel depositário, com vistas a respaldar 
o acesso aos relatórios, sendo fornecidas informações sobre o estudo e esclarecimentos acerca dos seus objetivos.

Após a assinatura do termo, deu-se início à segunda etapa do estudo, com a análise dos prontuários dos idosos atendidos pelo PAD com diagnóstico de LP. Posteriormente, solicitou-se ao setor de estatística um relatório com o levantamento referente ao período contemplado e aos custos associados ao tratamento de LP.Os dados foram inseridos num banco de dados utilizando o Microsoft Excel 2010, analisados estatisticamente, organizados em quatro planilhas divididas por período, procedimento, beneficiário e credenciado, e dispostos em tabelas.

Com a finalidade de observar os princípios éticos que norteiam a pesquisa envolvendo seres humanos, foram considerados nesta pesquisa os aspectos preconizados pelo Conselho Nacional de Saúde (CNS), de acordo com a Resolução 466/12, que incorpora referências básicas de pesquisa configurados em autonomia, não maleficência, beneficência e justiça.

\section{RESULTADOS}

Foram selecionados 158 beneficiários, que atenderam aos critérios de inclusão para o estudo. $\mathrm{O}$ total de indivíduos que recebeu atendimento em 2011 foi de 40 (25,32\%); em 2012, 48 (30,38\%) e, em 2013, 70 (44,30\%). No quesito referente aos custos totais, foi realizado um levantamento estatístico, observando-se que os valores desembolsados referentes aos tratamentos anuais foram de, respectivamente, $\mathrm{R} \$ 124.950,00 ; \mathrm{R} \$ 158.500,00$ e $\mathrm{R} \$ 179.021,48$ — dados apresentados na Tabela 1.

Tais atendimentos foram divididos de acordo com a demanda entre três equipes credenciadas em 2008, 2009 e 2013, respectivamente, que prestam serviço especializado à instituição e serão nomeadas neste estudo como $\mathrm{A}, \mathrm{B}$ e

Tabela 1. Custos por período/atendimento beneficiário, Fortaleza (CE), 2015.

\begin{tabular}{|c|c|c|c|}
\hline Período & 2011 & 2012 & 2013 \\
\hline $\begin{array}{l}\text { Atendimentos } \\
\text { beneficiários } \\
\text { com LP }\end{array}$ & 40 & 48 & 70 \\
\hline $\begin{array}{l}\text { Percentual de } \\
\text { atendimentos }\end{array}$ & 25,32 & 30,38 & 44,30 \\
\hline Custos (R\$) & $124.950,00$ & $158.500,00$ & $179.021,48$ \\
\hline Total (R\$) & $124.950,00$ & $158.500,00$ & $179.021,48$ \\
\hline
\end{tabular}

C. Essas subdividem os procedimentos executados por enfermeiras estomaterapeutas em três níveis, cujos valores foram acordados e definidos por processos contratuais. Os procedimentos são definidos como curativos pequeno, médio e grande, cujos valores, na época, eram, respectivamente, $\mathrm{R} \$ 85,00, \mathrm{R} \$ 95,00$ e $\mathrm{R} \$ 110,00$, e contemplavam material, medicamentos, custos operacionais e honorários, demonstrado nas Tabelas 2 e 3 .

Ao analisarmos os custos médios individuais por beneficiário (Tabela 4), esses resultados se refletem em um impacto econômico positivo para a empresa, que, no decorrer desse período, teve uma redução nos valores gastos. Em 2011, o

Tabela 2. Custos por empresa credenciada, Fortaleza (CE), 2015.

\begin{tabular}{lccc}
\hline Credenciado & $\mathbf{2 0 1 1}$ & $\mathbf{2 0 1 2}$ & $\mathbf{2 0 1 3}$ \\
\hline $\mathrm{A}(\mathrm{R} \$)$ & $124.950,00$ & $152.120,00$ & $110.416,48$ \\
\hline $\mathrm{B}(\mathrm{R} \$)$ & - & $6.380,00$ & $8.345,00$ \\
\hline $\mathrm{C}(\mathrm{R} \$)$ & - & - & $60.260,00$ \\
\hline Total $(\mathrm{R} \$)$ & $124.950,00$ & $158.500,00$ & $179.021,48$ \\
\hline
\end{tabular}

Tabela 3. Custos por procedimento, Fortaleza (CE), 2015.

\begin{tabular}{lccc}
\hline $\begin{array}{l}\text { Procedimento } \\
\text { Taxa de curativo }\end{array}$ & $\mathbf{2 0 1 1}$ & $\mathbf{2 0 1 2}$ & $\mathbf{2 0 1 3}$ \\
$\begin{array}{l}\text { pequeno }(\mathrm{R} \$) \\
\text { laxa de curativo }\end{array}$ & $51.440,00$ & $43.720,00$ & $43.789,00$ \\
$\begin{array}{l}\text { médio }(R \$) \\
\text { Taxa de curativo }\end{array}$ & $31.075,00$ & $55.580,00$ & $66.490,48$ \\
grande $(R \$)$ & $59.200,00$ & $68.742,00$ \\
\hline Total $(R \$)$ & $124.950,00$ & $158.500,00$ & $179.021,48$ \\
\hline
\end{tabular}

Tabela 4. Comparativo de custo anual total, Fortaleza (CE), 2015.

\begin{tabular}{lccc}
\hline Período & 2011 & 2012 & 2013 \\
\hline $\begin{array}{l}\text { Quantidade de } \\
\text { beneficiários } \\
\text { atendidos }\end{array}$ & 40 & 48 & 70 \\
$\begin{array}{l}\text { Custo total por } \\
\text { período (R\$) }\end{array}$ & $124.950,00$ & $158.500,00$ & $179.021,48$ \\
\hline $\begin{array}{l}\text { Custo médio } \\
\text { individual }(R \$)\end{array}$ & $3.123,75$ & $3.302,08$ & $2.557,44$ \\
\end{tabular}


custo médio por beneficiário era em torno de $\mathrm{R} \$ 3.123,75$, enquanto que, ao final de 2013, constatou-se que estava em torno de $\mathrm{R} \$ 2.557,45$.

\section{DISCUSSÃO}

A comunicação entre a instituição e os prestadores de serviço é realizada por meio do envio de relatórios quinzenais contendo registros fotográficos referentes às lesões que estão em tratamento. Nesse relatório é feita uma estimativa do tratamento a ser realizado durante o mês, constando número de visitas, local da lesão e especificação do tamanho do curativo, que é submetido à análise da auditoria de Enfermagem, a qual posteriormente emite parecer favorável ou não às solicitações.

Quanto à localização da LP, a maior ocorrência foi a região sacral, com 61,84\%, seguida de calcâneos, com 23,68\% do total, e trocantérica, com 14,47\%. O maior número de LP foi identificado entre os pacientes procedentes de internação hospitalar.

$\mathrm{Na}$ Itália, um estudo identificou que a prevalência de LP era de $18,0 \%$ e que, durante um seguimento de 12 meses, o grupo de pacientes com lesões tinha maior probabilidade de morte. $\mathrm{Na}$ Holanda, mais de 1,0\% do orçamento total do sistema de saúde é gasto na prevenção e no tratamento das LP, sendo este o quarto problema de saúde que mais consome recursos no país ${ }^{11}$.

Ressalta-se que todos os beneficiários participantes da amostra, na época, receberam visitas domiciliares de auditoria in loco, quando foram verificadas as condutas adotadas por cada equipe de profissional estomaterapeuta, as frequências das profissionais durante o tratamento e as percepções que os cuidadores/familiares tinham a respeito do tratamento e dos cuidados dispensados. Durante essas visitas, os cuidadores também receberam orientações referentes aos cuidados e à prevenção de LP, reforçando o trabalho das equipes prestadoras de serviço.

Ao longo desses 36 meses, foi possível constatar um aumento no número de beneficiários atendidos (de 40 para 70 , ou seja, 75,0\%) e, consequentemente, nos valores pagos (de $\mathrm{R} \$ 124.950,00$ para $\mathrm{R} \$ 179.021,48$ - 43,0\%), conforme a demanda/ano. Porém, por meio do comparativo anual (2011/2012 e 2012/2013), verificou-se que o custo total no primeiro biênio foi em torno de $26,0 \%$ do total da empresa, seguido por um custo gerado no segundo biênio de 13,0\%.
No Brasil, não existem dados precisos na literatura acerca dos custos gerados pelas LP para o sistema de saúde; porém, estudos realizados isoladamente fornecem dados que permitem a inferência de um panorama geral da situação ${ }^{14}$.

Estudo conduzido em Minas Gerais verificou que o tratamento das LP ocupa o terceiro lugar em gastos em saúde, sendo ultrapassado somente pelo tratamento de câncer e cirurgias cardíacas ${ }^{3-15}$.

Essa redução é atribuída ao trabalho educativo com um caráter preventivo desenvolvido periodicamente com eficiência pela equipe de auditoria externa da empresa estudada, cujo principal foco é proporcionar um atendimento de qualidade e excelência aos seus beneficiários associado à otimização dos custos com o tratamento.

\section{CONCLUSÃO}

A partir dos resultados obtidos, confirma-se que a LP é um problema de saúde atual e complexo, considerado de alto custo financeiro, que acomete facilmente idosos com síndrome da imobilidade, seja em ambiente hospitalar ou domiciliar.

Esse impacto financeiro gerado para a instituição encontra na prevenção uma forte aliada para o controle desses gastos, visto que é mais rentável investir em profissionais educadores do que na problemática já instalada.

Reforça-se que a Enfermagem deve intensificar os esforços para promover ações que esclareçam as dúvidas quanto ao tratamento e aos cuidados preventivos, valorizando os sentimentos de insegurança e dúvidas, tanto dos cuidadores quanto dos indivíduos acometidos.

Vale ressaltar que as inovações tecnológicas relacionadas a essa terapêutica, associadas a inúmeras medidas preventivas, são de grande importância para evitar o surgimento dessas lesões, cabendo aos profissionais de saúde a prática contínua do ato de educar.

Nesse contexto, é relevante a implantação de estratégias com caráter educativo sobre LP, incentivadas pelos profissionais de saúde envolvidos, e o monitoramento dos casos existentes, não somente voltado aos custos, mas também para a qualidade do serviço prestado, possibilitando uma melhor aplicabilidade dos recursos disponíveis, otimizando os custos e garantindo excelência no tratamento. 


\section{REFERÊNCIAS}

1. Morsch P, Pereira GN, Navarro JH, Trevisan MD, Lopes DG, Bós AJG. Características clínicas e sociais determinantes para o idoso sair de casa. Cad Saúde Pública. 2015;31(5):1025-34.

2. Barros MA, Sousa SMA, Costa ALB, Rosa LS, Jansen RCS. Incidência e prevalência de úlceras por pressão em pacientes internados em um hospital universitário. Rev Estima. 2013;11(4):17-24.

3. Silva AJ, Pereira SM, Rodrigues A, Rocha AP, Varela J, Gomes LM, et al. Custo econômico do tratamento das úlceras por pressão: uma abordagem teórica. Rev Esc Enferm USP. 2013;47(4):971-6.

4. Pedrosa IL, Silva MS, Araújo AA, Schwanke CH, DeCarli GA, Gomes I. Úlceras por pressão em idosos e não idosos: estudo de coorte histórica. Online Braz J Nurs. 2014;13(1):82-91.

5. Sarquis MG. Orientações para a prática clínica no tratamento e prevenção de úlceras por pressão. In: Malagutti W, Kakihara CT, organizadores. Curativos, estomias e dermatologia: uma abordagem multiprofissional. São Paulo: Martinari; 2010. p. 223-32.

6. Freitas MC, Medeiros AB, Guedes MV, Almeida PC, Galiza FT, Nogueira JM. Lesão por pressão em idosos institucionalizados. Rev Gaúcha Enferm. 2011;32(1):143-50.

7. Santos CT, Oliveira MC, Pereira AG, Suzuki LM, Lucena AF. Indicador de qualidade assistencial lesão por pressão: análise de prontuário e de notificação de incidente. Rev Gaúcha Enferm. 2013;34(1):111-8.

8. Lima AC, Guerra DM. Avaliação do custo do tratamento de úlceras por pressão em pacientes hospitalizados usando curativos industrializados. Ciênc Saúde Coletiva. 2011;16(1):267-77.

9. Montesinos MJ. Programa de gestão da qualidade assistencial: uma oportunidade de melhoria no procedimento de curativo. In: Malagutti W, Kakihara $\mathrm{CT}$, organizadores. Curativos, estomias e dermatologia: uma abordagem multiprofissional. São Paulo: Martinari; 2010. p. 201-21

10. Silva KL, Sena RR, Silva PM, Braga PP, Souza CG. Serviços de atenção domiciliar na saúde suplementar e a inserção da enfermagem em Belo Horizonte/MG. Acta Paul Enferm. 2012;25(3):408-14.

11. Chayamiti EM, Caliri MH. Lesão por pressão em pacientes sob assistência domiciliária. Acta Paul Enferm. 2010;23(1):29-34.

12. Coêlho AD, Lopes MV, Melo RP, Castro ME. O idoso e a lesão por pressão em serviço de atendimento domiciliar. Rev Rene. 2012;13(3):639-49.

13. Hyeda A, Costa ES. Uma análise preliminar dos custos em quimioterapia ambulatorial no sistema de saúde suplementar. J Bras Econ Saúde. 2015;7(2):99-109.

14. Soares DA, Vendramin FS, Pereira LM, Proença PK, Marques MM. Análise da incidência de úlcera de pressão no Hospital Metropolitano de Urgência e Emergência em Ananindeua, PA. Rev Bras Cir Plast. 2011;26(4):578-81.

15. Freitas JP, Alberti LR. Aplicação da Escala de Braden em domicílio: incidência e fatores associados a lesão por pressão. Acta Paul Enferm. 2013;26(6):515-21. 